\title{
ON THE ACCRETIVITY OF THE INVERSE OF AN ACCRETIVE RELATION
}

\section{GUSTAF GRIPENBERG}

\begin{abstract}
If $X$ is a smooth, reflexive, real Banach space such that a relation $A$ in $X \times X$ is accretive iff $A^{-1}$ is accretive, then $X$ is isomorphic to a Hilbert space.
\end{abstract}

Let $X$ be a real Banach space with dual $X^{*}$. A subset $A \subset X \times X$ is said to be accretive if for all $\left[x_{i}, y_{i}\right] \in A, i=1,2,\left(y_{1}-y_{2}, f\right) \geqslant 0$ for some $f \in$ $F\left(x_{1}-x_{2}\right)$, where $F$ is the duality map: $F(x)=\left\{x^{*} \in X^{*} \mid\left(x, x^{*}\right)=\|x\|^{2}=\right.$ $\left.\left\|x^{*}\right\|^{2}\right\}$. By definition $A^{-1}=\{[x, y] \mid[y, x] \in A\}$. It is trivial that if $X$ is a real Hilbert space then $A$ is accretive (or monotone) iff $A^{-1}$ is accretive.

Theorem. Suppose $X$ is a smooth, reflexive, real Banach space such that $A$ is accretive in $X \times X$ iff $A^{-1}$ is accretive in $X \times X$. Then $X$ is isomorphic to a Hilbert space.

Proof. As $X$ is smooth and reflexive, the duality map $F$ is single-valued. Define $\langle x, y\rangle=(x, F(y)), x, y \in X$. It follows from the assumption that $\langle x, y\rangle \geqslant 0$ iff $\langle y, x\rangle \geqslant 0$, and so also that

$$
\langle x, y\rangle=0 \Leftrightarrow\langle y, x\rangle=0 \quad \forall x, y \in X .
$$

For any closed subspace $M$ of $X$ define

$$
M^{\perp}=\{x \in X \mid\langle y, x\rangle=0 \forall y \in M\} .
$$

By (1) it is seen that $M$ is a closed subspace of $X$. If $x \in M \cap M^{\perp}$ then $\|x\|^{2}=\langle x, x\rangle=0$, i.e., $x=0$; and so

$$
M \cap M^{\perp}=\{0\} .
$$

Let $z \in X$ be arbitrary. Let $x_{1}$ be an element in $M$ that minimizes the norm $\|z-x\|, x \in M$ ( $x_{1}$ exists because $M$ is closed and convex and $X$ is reflexive). This implies that $\left\|z-x_{1}\right\| \leqslant\left\|z-x_{1}+y\right\| \forall y \in M$. Lemma 1.1 in [1] now yields $\left\langle y, z-x_{1}\right\rangle=0 \forall y \in M$, and so $z-x_{1} \in M^{\perp}$. It follows that

$$
X=M+M^{\perp}
$$

which together with (3) and the fact that $M^{\perp}$ is a closed subspace shows that $M$ has a topological complement. As $M$ was an arbitrary closed subspace of $X$, Theorem 1 in [2] implies that $X$ is isomorphic to a Hilbert space.

Received by the editors September 13, 1976.

AMS (MOS) subject classifications (1970). Primary 46C10, 47H05.

C American Mathematical Society 1977 


\section{REFERENCES}

1. T. Kato, Nonlinear semigroups and evolution equations, J. Math. Soc. Japan 19 (1967), 508-520. MR 37 \# 1820.

2. J. Lindenstrauss and L. Tzafriri, On the complemented subspaces problem, Israel J. Math 9 (1971), 263-269. MR 43 \#2474.

Institute of Mathematics, Helsinki University of Technology, SF-02150 EspoO 15, FINLAND 